\title{
High Voltage DC Generation using Marx Generator
}

\author{
Gaytri Saraf $^{1}$, Amrapal Bansode ${ }^{2}$, Akshada Khule ${ }^{3}$, Shruti Rangari ${ }^{4}$, Prof. S.U. Shinde ${ }^{5}$ \\ Bachelor of Engineering, Electrical Engineering, ZCOER, Pune, India ${ }^{1,2,3,4}$ \\ Professor, Electrical Engineering, ZCOER, Pune, India ${ }^{5}$
}

\begin{abstract}
The main aim of the project proposes high voltage DC generation using Marx generator precept that uses MOSFET alongside capacitor stacks. The Marx precept turned into brought with the aid of Erwin Otto Marx. The principle includes producing a pulse of excessive voltage with the aid of the usage of some of capacitors parallely in an effort to charge up to on time after which installation in connection collection to develop an excessive voltage for the duration of the off length. The machine is used inside the method of era of in Kv's for actual time checking out to be finished on electrical equipment insulation effectiveness to be placed up on strength carriers. The system includes 4 stages such that everyone is made up from diodes, one MOSFET in conjunction with one capacitor. Diodes are used in capacitor charging at every stage and MOSFET is used as a transfer to keep away from power losses. The capacitors are charged in parallel in the course of the ON time using 555 timer. The capacitors are delivered in a sequence the use of MOSFET switches in the course of the OFF time. Our challenge offers a compact and smooth device from a DC deliver of $12 \mathrm{~V}$ to get an approx (36-40) Volts.
\end{abstract}

Keywords: IC555, Opto coupler, MOSFET, Marx generator.

\section{INTRODUCTION}

Rapid discharge of stored energy in short interval as electrical pulses into a load produces big quantity of instant power. The traits of pulse as rise time, fall time, pulse width, repetition charge, a voltage and strength tier varies with unique applications. High voltage pulsed electricity have extensive variety of programs in exclusive fields like industrial, scientific, agricultural, environmental etc. Marx Generator is an excessive voltage pulse generator. The essential precept of Marx Generator is that the capacitors are charged in parallel as much as its input DC voltage stage. Those capacitors are then related in series the use of switches to produce an excessive voltage pulse throughout the burden technology. With the improvement of solid state electronics, solid-state devices have become more and more appropriate for pulsed power application. They might offer the pulsed energy systems with compactness, reliability, excessive repetition fee, and lengthy existence time. The rising of pulsed electricity generators the usage of solid-state gadgets gets rid of barriers of traditional additives, and promises pulsed strength era to be extensively utilized in business packages. However, strong-state switching devices inclusive of MOSFET to be had now are best rated up to 3 kilo volts. Maximum of pulsed power systems demand of much higher voltage rating.

Switching devices are vital additives in pulsed power systems. Conventional Marx Generator use spark gas switches. These switches possess barriers like short lifestyles time in terms of number of operation cycles, low switching frequency, huge length, extra maintenance and many others. In current years the strong country switches like MOSFET or IGBT is utilized in place of spark gaps. The benefits of solid country switches are compact, reliable, bendy, more efficient, long lifestyles time, low charges and reduced losses. The output pulse width and amplitude each may be varied by controlling the gate control pulses to the switches.

Both MOSFET and IGBT give variable pulse width and can generate a fast pulse rise time within a few ns across the load. The switching time of MOSFET is much lesser than IGBT (typically:- $20 \mathrm{~ns}$ for a MOSFET, $200 \mathrm{~ns}$ for an IGBT). IGBT are available at higher voltage ratings (up to $6500 \mathrm{~V}$ ), where as the MOSFETs are limited to $1200 \mathrm{~V}$.

Solid state Marx Generator has replaced the charging resistors in conventional Marx with high voltage diodes. Many new topologies with solid state Marx Generators are proposed in literature. Yifan Wu has proposed repetitive and high voltage Marx Generator using solid-state devices with inductive based charging. Inductor here acts as a current limiter at the time of pulse generation. Drawback of this topology is that it limits the pulse frequency due to long charging time constant. Also it provides no isolation from input supply source during discharging mode. A novel solid-state pulsed power modulator driving by magnetic ring transformers is introduced by Jian Qiu. The charging power supply here is a full bridge resonant inverter. There is provision of isolation between charging and discharging loops implemented by magnetic rings. But the limitation is that due to the use of magnetic rings, the stray parameters distort the output voltage waveforms. Ju Won Baek proposed a novel repetitive impulse voltage generator of unipolar configuration by using a 
boost converter array. The circuit can easily obtain a high voltage pulse without pulse transformer. The proposed circuit allows operation at kilohertz frequency with high efficiency. The drawback is that if the number of boost stacks increases the line inductance increases and hence the rise time of the output pulse increases. L.M. Redondo have proposed a topology of solid state Marx Generator with energy recovery reset circuit using transformer connected at the output. This scheme provides galvanic isolation to the load. But a limitation is that leakage inductance of transformer limits the rise time of output pulse. The efficiency of the system decreases due to losses and size of transformer.

\section{PROPOSED METHOD}

The Marx precept changed into advanced by way of Erwin Otto Marx. Its precept is to generate an excessive voltage pulse. The use of some of capacitors in parallel to charge up during the on time after which connected in collection to increase higher voltage during the off length. This precept is used to generate voltages inside the range of KV's in actual-time for testing the insulation of the digital home equipment like transformers and the insulation of the power carrying lines. This task includes 4 degrees and each degree is manufactured from one MOSFET, two diodes, and one capacitor. MOSFET is used as a switch; diodes are used to charge the capacitor at every degree without power loss. A 555 timer generates pulses for the capacitors to charge in parallel during ON time. During OFF time of the pulses the capacitors are brought in series with the assist of MOSFET switches. Subsequently, wide variety of capacitors utilized in series (four in our challenge) adds up the voltage to approximately 3 (4 capacitors-1 capacitor) instances the supply voltage. This device shape gives compactness and easiness to make entire system.

\section{BLOCK DIGRAM}

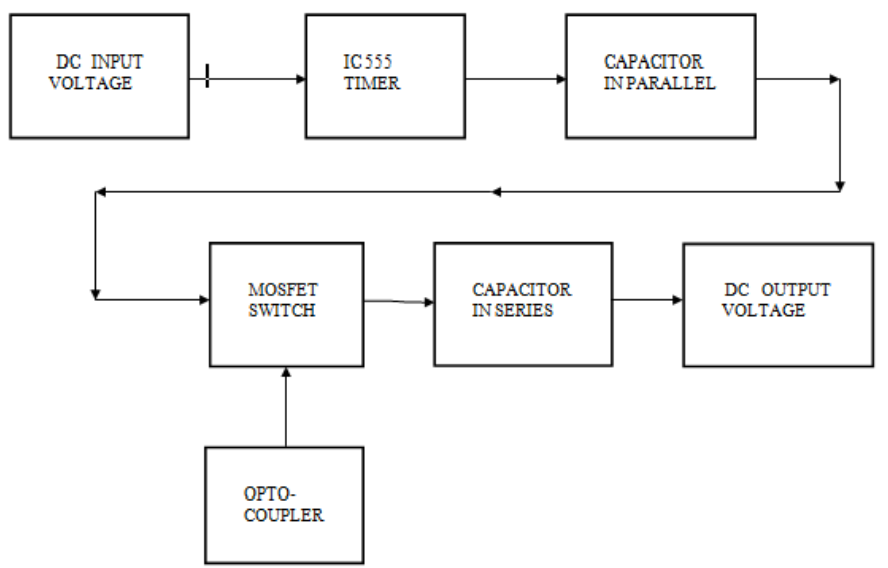

Fig.1 Block Diagram of Marx generator

Primary energy supply is taken as a step down AC supply. Its means step down to suitable voltage and rectified to get consistent DC supply for charging of capacitors. Capacitors are charge storage tool. The charging of capacitor takes place as they are parallel linked to the rectifier. When capacitor is having suitable charge saved in it, switches are used to attach all capacitor in series and discharge of capacitor take region and we get $\mathrm{n}$ times of rectifier voltage across the burden. Because of numerous sensible constraints, the output voltage is incredibly much less than $n \times V$ (in which $n$ is a degree).

\section{HARDWARE}

\section{Conventional Marx Generator}

The generator capacitance $\mathrm{C}$ is to be first charged and then discharged into the wave shaping circuits. A single capacitor $\mathrm{C}$ may be used for voltages as much as 2 hundred $\mathrm{kV}$. For generating very excessive voltages, bank of capacitor are charged in parallel and then discharged in series. The association for charging the capacitors in parallel after which connecting them in series for discharging was at first proposed with the aid of Erwin Otto Marx in 1923 as proven in Fig.1. usually the charging resistance is selected to restrict the charging modern to about 50 to $100 \mathrm{~mA}$, and the generator capacitance $\mathrm{C}$ is selected such that the product CRs is about 10s to $1 \mathrm{~min}$. the gap spacing is selected such that the breakdown voltage of the distance $G$ is greater than the charging voltage $V$. accordingly, all of the capacitances are charged to the voltage $\mathrm{V}$ in about 1 minute. When the impulse generator is to be discharged, the gaps $\mathrm{G}$ are made to spark over simultaneously by way of a few outside approaches. Hence, all the capacitors $\mathrm{C}$ get connected in series and discharge into the weight capacitance or the take a look at item. The discharge time steady CR1/n (for n stages) will be 
very small in comparison to charging time steady CRs a good way to be few be very small be very small as compared to charging time steady CRs for you to be few seconds.

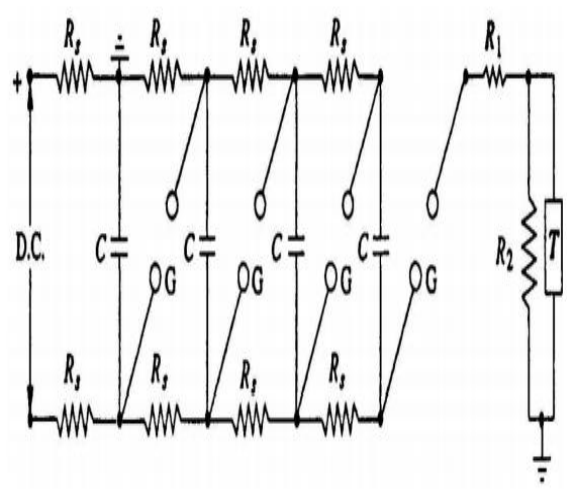

Fig. 2 Conventional Marx generator

There are some demerits inside the traditional Marx circuit as follows:

1. Long charging time because the charging cutting-current flows through the charging resistors.

2. Low efficiency due to the identical cause referred to above.

3. Low repetition rate.

4. Few output voltage appearance in charging duration because the charging modern flows via the charging resistors and a load.

5. Turn-off is not possible due to the usage of the spark gap switches.

That allows you to clear up those troubles, a few new Marx circuits are proposed. Those new progressed circuits use a semiconductor switch which includes MOSFETs.

\section{Modern Marx Generator}

The generator capacitance $\mathrm{C}$ is to be first charged after which discharged into the wave shaping circuits. A single capacitor $\mathrm{C}$ may be used for voltages as much as two hundred $\mathrm{kV}$. For generating very excessive voltages, banks of capacitor are charged in parallel after which discharged in series. The association for charging the capacitors in parallel and then connecting them in collection for discharging became initially proposed by using Erwin Otto Marx in 1923 as proven in Fig.3. Normally the charging resistance is selected to restrict the charging current to approximately 50 to 100 $\mathrm{mA}$, and the generator capacitance $\mathrm{C}$ is chosen such that the product $\mathrm{CRs}$ is ready 10 s to at least one min. The space spacing is chosen such that the breakdown voltage of the distance $\mathrm{G}$ is more than the charging voltage $\mathrm{V}$. As a result, all of the capacitances are charged to the voltage $\mathrm{V}$ in about 1 minute. While the impulse generator is to be discharged, the gaps $\mathrm{G}$ are made to spark over concurrently by using some external means. For that reason, all the capacitors $\mathrm{C}$ get related in series and discharge into the load capacitance or the check item. the discharge time constant CR1/n (for $n$ tiers) will be very small as compared to charging time constant CRs so that it will be few be very small be very small compared to charging time steady CRs if you want to be few seconds.

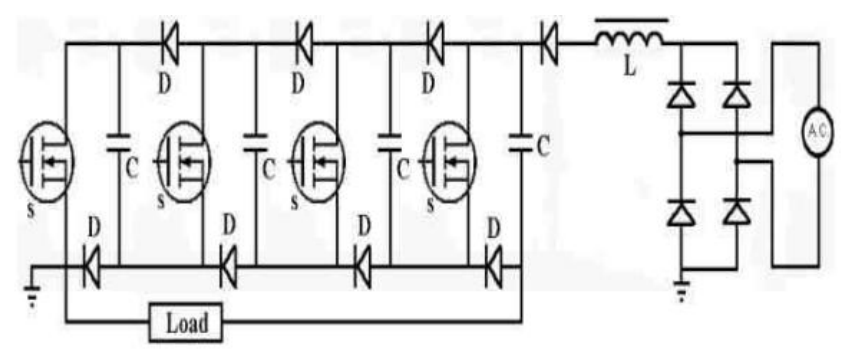

Fig.3 Modern Marx Generator

\section{DESIGN CALCULATIONS}

$\mathrm{C}=(\mathrm{t} * \mathrm{Vo}) /((20 \%$ of $\mathrm{Vo}) * \mathrm{R})$

Where,

$\mathrm{Vo}=$ Output voltage,

$\mathrm{t}=$ maximum pulse width. 
$\mathrm{R}=$ Resistance connected at load side.

Assuming 10-21\% drop in output voltage.

$\mathrm{C}=\left(20 \mathrm{msec}^{*} 48\right) /((48 * 21 \%) * 3900)=24.42 \mu \mathrm{F}$

$\mathrm{Ceq}=\mathrm{n} * \mathrm{C}=4 *(24.42 \mu \mathrm{F})=97.6800 \mu \mathrm{F} \sim=100 \mu \mathrm{F}$.

- $\quad$ Resistors $-1 \mathrm{k}, 10 \mathrm{k}, 3.9 \mathrm{k}$

- $\quad$ [range(10-100k);max voltage(50-100kV)]

- $\quad$ MOSFET - IRFZ44

- $\quad$ Diode - 1N4007

- Opto-coupler - MCT2E

\section{RESULTS}

We give the input voltage $12 \mathrm{~V}$ and at the output stage we get the output $41.2 \mathrm{~V}$. The output voltage is depends on the no. of stages which are used in circuit. If we increase the no. of changes then the output voltage will change.

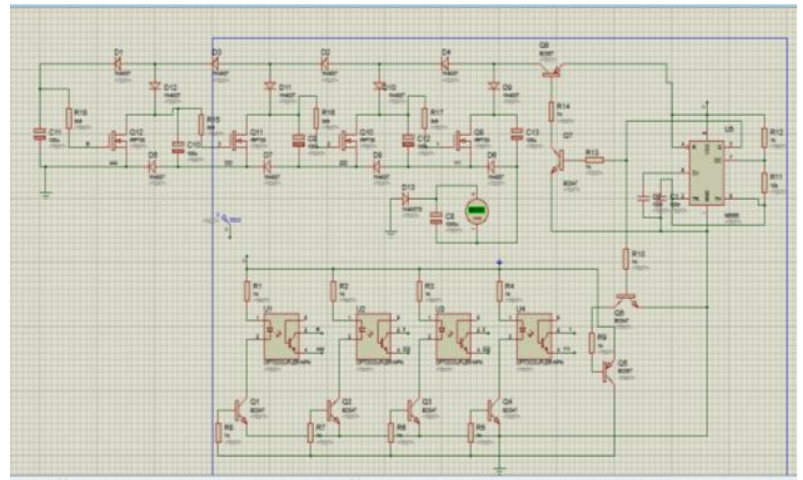

Fig. 4 Simulation result

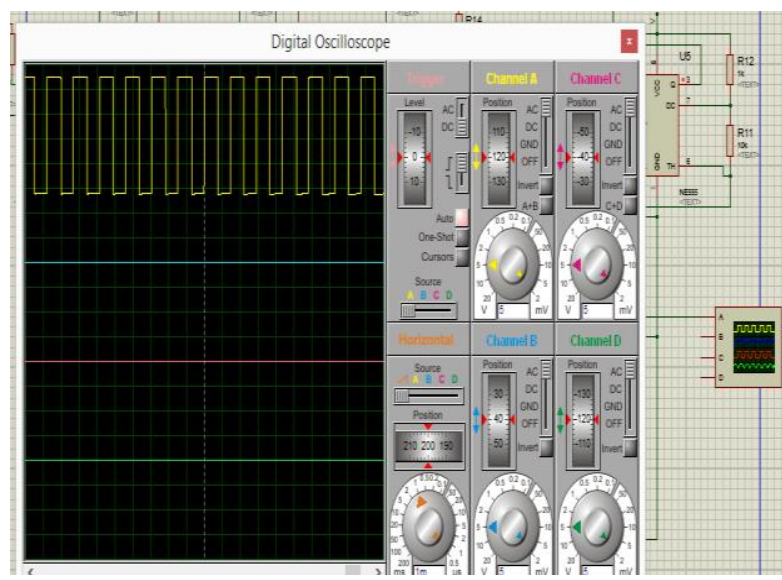

Fig.5 Output waveform on oscilloscope

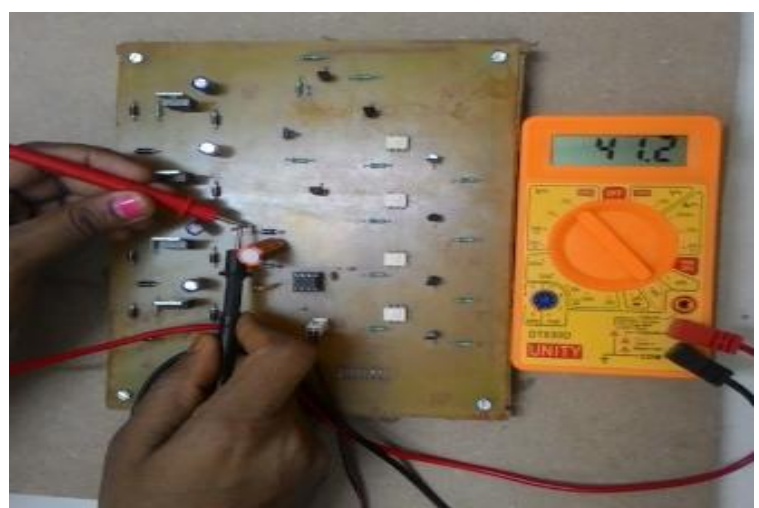

Fig.6 Output of high voltage Marx generator 


\section{CONCLUSION}

The simulation gives the concept of HVDC generation i.e., $2 \mathrm{kV}$ the use of sphere gaps. In this study, solid state devices consisting of MOSFET and diodes are used in Marx generator to replace of switches and resistors. Moreover, its miles reasonable that MOSFET drivers make use of method of self-provided power. The Marx generator is used to multiply voltage by way of the usage of MOSFETS. The number of MOSFETS used comes to a decision the wide variety of times the voltage needs to be increased. In this examine we've got used four stages in hardware and the circuit multiplies the enter voltage effectively.

\section{FUTURE SCOPE}

The output voltage increment depends on the amount of stages. The switching devices i.e., MOSFETS is replaced by way of the alternative devices. In this circuit we obtain high voltage and low current so in future we increase voltage as well as current.

\section{REFERENCES}

[1] J.W.Baek, D.W. Yoo, G.H. Rim, and J.S. Lai, " Solid State Marx generator using series connected IGBTs Il, IEEE Trans. on Plasma Science, Vol. 33, No. 4, pp.1198-1204, Aug. 2005.

[2] Kefu Liu, Yan Luo and Jian Qiu, "A Repetitive High Voltage Pulse Adder Based on Solid State Switches", IEEE Trans. on Dielectrics and Electrical Insulation Vol. 16, No. 4, August 2009.

[3] Tatsuro Sakamoto and Hidenori Akiyama, "Solid-State Dual Marx Generator With a Short Pulse width", IEEE Trans. on Plasma Science, Vol. 41, No. 10, October 2013.

[4] Harshada C. Bhosale, Bindu S., Sincy G., P.C. Saroj, Archana S, " Design and Simulation of 50 kV, 50 A Solid State Marx Generator", IEEE, International Conference on Magnetics, Machines \& Drives (AICERA- 2014 iCMMD).

[5] "Solid State Pulsed Power Systems", Dr. Stephan Roche Physique \& industrie, 17 rue de la rente Logerot, 21160 Marsannay la cote, France.

[6] R. Cassel and S. Hitchcock, -A new type high voltage fast rise/fall time solid state Marx pulse modulatorll, IEEE Particle Accelerator Conf., New Mexico, USA, pp. 865-867, 2007.

[7] Yifan Wu, Kefu Liu, Jian Qiu , XiaoXu Liu and Houxiu Xiao, " Repetitive and High Voltage Marx Generator Using Solid-state Devices", IEEE Trans. on Dielectrics and Electrical Insulation Vol. 14, No. 4; August 2007.

[8] Jian Qiu, Kefu Liu and Liuxia Li, " Stray Parameters in a Novel Solid State Pulsed Power Modulator", IEEE Trans. on Dielectrics and Electrical Insulation Vol. 20, No. 4; August 2013.

[9] Ju Won Baek, Dong Wook Yoo, Geun Hie Rim and Jih-Sheng (Jason) Lai, "Solid State Marx Generator Using Series- Connected IGBTs", IEEE Trans. on Plasma Science, Vol. 33, No. 4, August 2005.

[10] L.M.Redondo, J.Fernando Silva, P.Tavares and E.Margato, "Solid-state Marx generator design with an energy recovery reset circuit for output transformer association".

[11] Tatsuro Sakamoto, Alireza Nami, Masahiro Akiyama, and Hidenori Akiyama, " A Repetitive Solid State Marx-Type Pulsed Power Generator Using Multistage Switch-Capacitor Cells", IEEE Trans. on Plasma Science, Vol. 40, No. 10, October 2012. 